\title{
Research on the management of multimedia online education based on the virtual architecture of network
}

\author{
function \\ TANG Li Li ${ }^{1}$,TANG $\mathrm{Li}^{2}$ \\ \{TangLiLi3812@163.com¹,TangLi3812@163.com²\} \\ 1,2 Jiangxi Institute of Applied Science and Technology, Nanchang 330100, China;
}

\begin{abstract}
The traditional multimedia online education management method can not effectively recommend the learning content that students need, which leads to poor learning effect and no obvious improvement of students' performance after learning. Therefore, a multimedia online education management method based on the virtual structure of network function is designed. Firstly, the online education resource learning recommendation model is constructed, the student attribute eigenvalues are extracted, and then the multimedia online education database is generated. Finally, the multimedia online education management system is established to complete the multimedia online education management based on the network function virtualization architecture. The experimental results show that the students' learning performance is higher than that of the traditional management method after learning the multimedia online education management method based on the network function virtualization architecture, which proves that the design of the multimedia online education management method based on the network function virtualization architecture can improve the students' learning effect.
\end{abstract}

Keywords: network function virtualization architecture; multimedia; online education; management; evaluation;

\section{Introduction}

With the development of Internet technology, the education mode has been upgraded gradually. In recent years, multimedia online education has developed rapidly. The characteristics and advantages of multimedia online education are two-way interaction, that is, through information technology to achieve the interaction and exchange between human and human, which can not only strengthen the communication between teachers and students, but also encourage and promote students' self-study and cooperation between students. Modern information technology provides multimedia online education with learning resources and learning environment that are more conducive to students' construction, multimedia online education breaks the traditional education model of "school attendance", overcomes the limitations of traditional education in time, space, age and economic conditions, pushes 
education to the front of the educated, while students pull education resources to their front through the network, according to the needs of colleges and universities, majors, courses and teachers, make their own teaching plans and arrangements to make teaching and learning more flexible and personalized. The position of multimedia online education in higher education system has risen to the main force of mass education and life-long education, becoming the first choice for governments to realize life-long education, and the development of educational technology characterized by knowledge and intelligence, which is a new trend in the development of education in the world. Multimedia online education with various media as the means of communication has input a continuous stream of human blood and knowledge resources for the development of human society. This kind of education method is favored by governments and enterprises all over the world because of its flexible means, continuous tracking of modern scientific and technological achievements, and direct transformation of those cutting-edge science and technology with application prospects into productivity. The traditional online education management method does not fully consider the content that users need to learn, and the performance after management has not been significantly improved, therefore, it is of great significance to design a multimedia online education management method based on the virtual structure of network functions to help analyze the learning mode of online course users and develop the most appropriate teaching evaluation system.

\section{Online education resource learning recommendation model}

The design of multimedia online education management method based on network function virtualization architecture [1] mainly provides personalized courses for learners to improve the effect of education management. The mechanism of multimedia online education management is to judge the current students' learning needs through the virtual architecture of network functions, and to provide appropriate personalized learning resources and learning support services with the help of recommendation technology. According to the rules of online learning and personalized learning needs of users, the collection, analysis and judgment of individual characteristics of learners are the premise of the implementation of personalized learning system recommendation mechanism. In this paper, the user learning style is tested by using the style scale, and then the test results are recorded in the user model database. Learning resource database is mainly used to store learning related materials, including courseware, video, audio and other electronic resources. Teaching strategy database is used to store some process learning activity templates designed by teachers, such as collaborative learning, inquiry learning, project learning, etc. During the operation of the system, we need to use the recommendation technology to extract the required information from the database, and then through the analysis and transformation of the program algorithm, the content of the 
resources that meet the current learners' individual needs will be presented in turn, so as to achieve the dynamic recommendation effect of resource personalization. The overall architecture is shown in Figure 1.

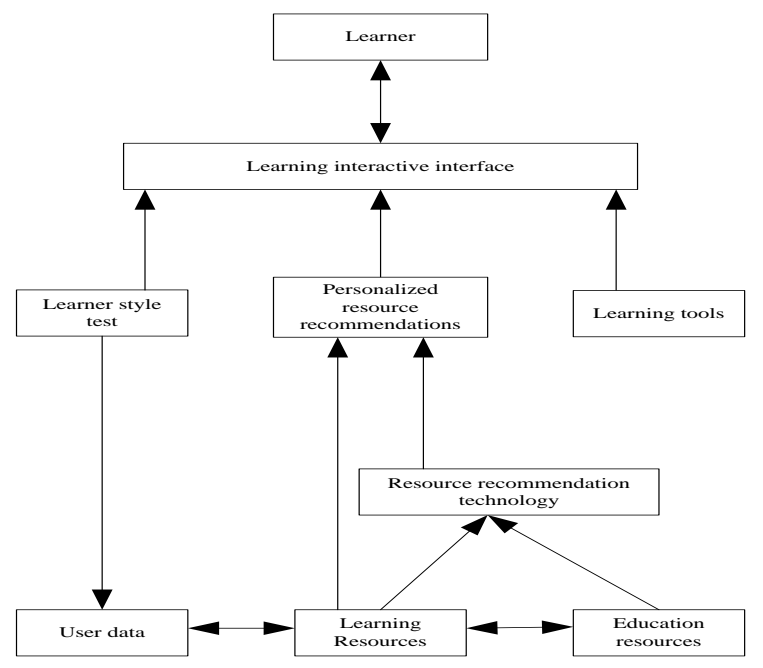

Fig.1. Personalized learning architecture based on network functional virtualization architecture When learners enter the website for the first time, they need to fill in basic personal information to complete the registration, mainly including name, gender, login name, login password, professional background and other basic information. At the same time, under the guidance of the system, learners need to use the learning style test module to evaluate their own learning style type. The user application process is shown in Figure 2

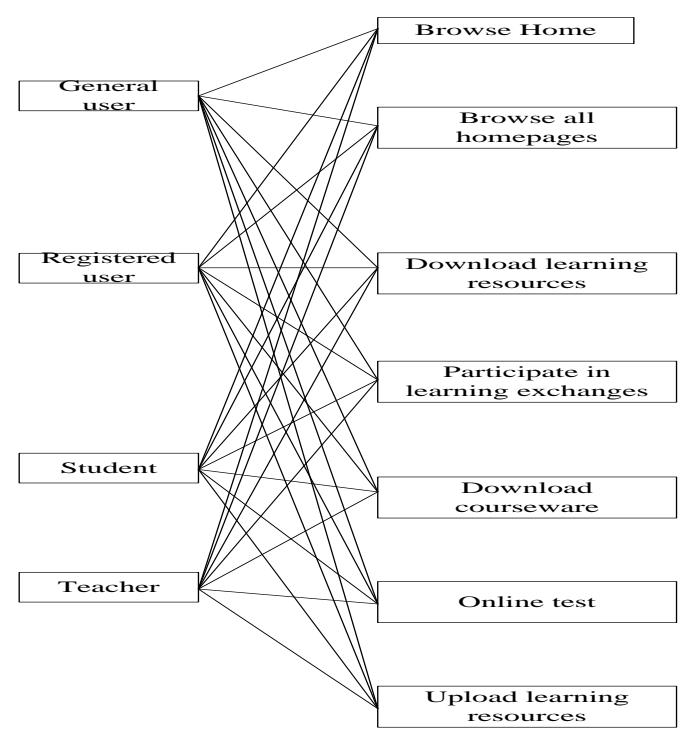


Fig.2. User Application Process

The learner modeling component processes the information and stores it in the user model database. In the process of learning, we can calculate and record the course resources learned by learners in real time by recorder, and update the learning record data at any time; At the same time, the resource recommendation mechanism needs to extract the current user's personal information from the user model database to determine the type of learning style, and use the recommendation algorithm to extract the dynamic recommendation of resources matching the user's learning style from the resource database and the teaching strategy database. The recommended process of collaborative filtering is as follows:

Step 1: fill in the missing value of the evaluation score matrix of the user knowledge object according to the requirements of the content filtering algorithm [3], and set the initialization score of the knowledge unit without evaluation to 0 ;

Step 2: build a user model based on the four dimensions of user's basic information, learning style, cognitive level and learning record, and calculate the similarity of the target user. The calculation formula is:

$$
f=\sum_{c} d / \frac{m}{g}
$$

In formula (1), $f$ represents the user basic information, $\sum_{c} d$ represents the user similarity calculation parameter, $g$ represents the information for the website, $m$ represents the learning basic content.

Step 3: Calculate the average score for all users as the cluster center. Calculate the distance between the current target user and the cluster center, and find out all other virtual user sets in the class to which the target user belongs.

Step 4: calculate the evaluation score of each knowledge object by calling the algorithm of knowledge content characteristic attribute value matrix. The calculation formula is:

$$
b=m / \frac{s}{\sqrt{c / i}} \text { (2) }
$$

In formula (2), $b$ represents the user to learn the knowledge content, $m$ represents the user to measure the score calculation factor, $\sqrt{c / i}$ represents the knowledge content characteristic attribute, $s$ represents the knowledge object appraisal index.

Step 5: judge whether the target user conducts knowledge unit evaluation, and if the 
result is yes, switch to the next step;

Step 6: calculate the similarity of all users in the target user according to the basic characteristics of the user (background information);

Step 7: calculate the similarity between the target user and all users in the website according to the score of knowledge unit evaluation;

Step 8: calculate the neighbor user set of the current target user according to the similarity ranking;

Step 9: according to the similarity ranking calculation, recommend the knowledge object list to the target user.

Learners use the auxiliary support service tools provided by the website (such as learning notebook, player, communication tools, etc.) for online personalized learning. At the end of learning, learners can learn about self-study through the unit test module provided by the website, and their test scores will also be recorded in the user model database in real time as the basis for judging learners' cognitive level when dynamically recommending resources. Recommendation technology is the core of realizing intelligent effect of personalized learning system, and it also embodies the recommendation idea of the system.

\section{Realization of multimedia online education management in virtualization architecture}

\subsection{Student attribute eigenvalue extraction}

On the basis of the above-mentioned online education resource learning recommendation model, for the multimedia online education management of the virtualization architecture, first extract the student attribute eigenvalue [4]. The traditional method of extracting the characteristic value of students' attributes is mainly based on the examination of students' achievements and whether the teachers have completed the teaching tasks. This evaluation method is relatively simple and does not fully consider the participation of the teaching subject, so it cannot accurately evaluate the teaching effect, let alone be used as the evaluation method of online courses. Online course is different from traditional classroom. Students who participate in online course leave a lot of data. By analyzing the user data of online course, the definition of student attribute is put forward, that is, the nature or behavior characteristics of students themselves or objectively. In short, it is the objective performance of students in a specific aspect. Based on the analysis of online course user data, this study summarizes the following student attributes and gives them characteristic values, which can be used in online learning modeling of teaching evaluation. It mainly includes the following aspects:

First, the student's gender, if the student's gender is male, the eigenvalue is 1 ; if the student's gender is female, the eigenvalue is 2 ; 
Second, classroom activity, its classification and characteristic values are shown in Table 1.

Table 1. Class Activity Characteristics

\begin{tabular}{ccc}
\hline Activity & Classification basis & Eigenvalues \\
\hline Highly active & Class participation $>200 \%$ & 1 \\
More active & $100 \%$ <class participation $<200 \%$ & 2 \\
Moderately active & $20 \%$ <class participation $<100 \%$ & 3 \\
Low activity & $0 \%<$ Class participation $<20 \%$ & 4 \\
Inactive & Class participation $=0 \%$ & 5 \\
\hline
\end{tabular}

Third: Forum activity, its classification and eigenvalues are shown in Table 2.

Table 2. Characteristics of Forum Activity

\begin{tabular}{ccc}
\hline Activity & Classification basis & Eigenvalues \\
\hline Highly active & Forum response rate $>200 \%$ & 1 \\
More active & $100 \%<$ forum response rate $<200 \%$ & 2 \\
Moderately active & $20 \%<$ forum response rate $<100 \%$ & 3 \\
Low activity & $0 \%<$ forum response rate $<20 \%$ & 4 \\
Inactive & Forum response rate $=0 \%$ & 5 \\
\hline
\end{tabular}

Fourth: job quality, its classification and eigenvalues are shown in Table 3.

Table 3. Job Quality Characteristics

\begin{tabular}{ccc}
\hline Operating quality & Classification basis & Eigenvalues \\
\hline high quality & Timely price increase, excellent rate> 90\% & 1 \\
Higher quality & $70 \%$ <excellent rate $<90 \%$ & 2 \\
Medium quality & $20 \%$ <excellent rate $<70 \%$ & 3 \\
Low quality & $0 \%$ <excellent rate $<20 \%$ & 4 \\
No quality & Excellent rate $=0 \%$ & 5 \\
\hline
\end{tabular}

Fifth: the degree of autonomous learning. The degree of autonomous learning mainly determines the positive degree of students'pre-study and the degree of participation in review after class [5].

One of the after-class questions with the classroom activity and forum activity part of the coincidence, so no longer set properties. According to the above process, the extraction of eigenvalues of students'attributes is completed, which provides the basic significance for the multimedia online education management of virtualization architecture.

\subsection{Multimedia online education database generation}

In order to improve the speed of data response, multimedia online education database is designed. The database of this design is mainly composed of three parts. The first part is the 
standardization of data processing, the second part is data mining analysis and integration [6], the third part is the database. The core of the middle tier is the second part, which is also the core of online education management, as shown in Figure 3.

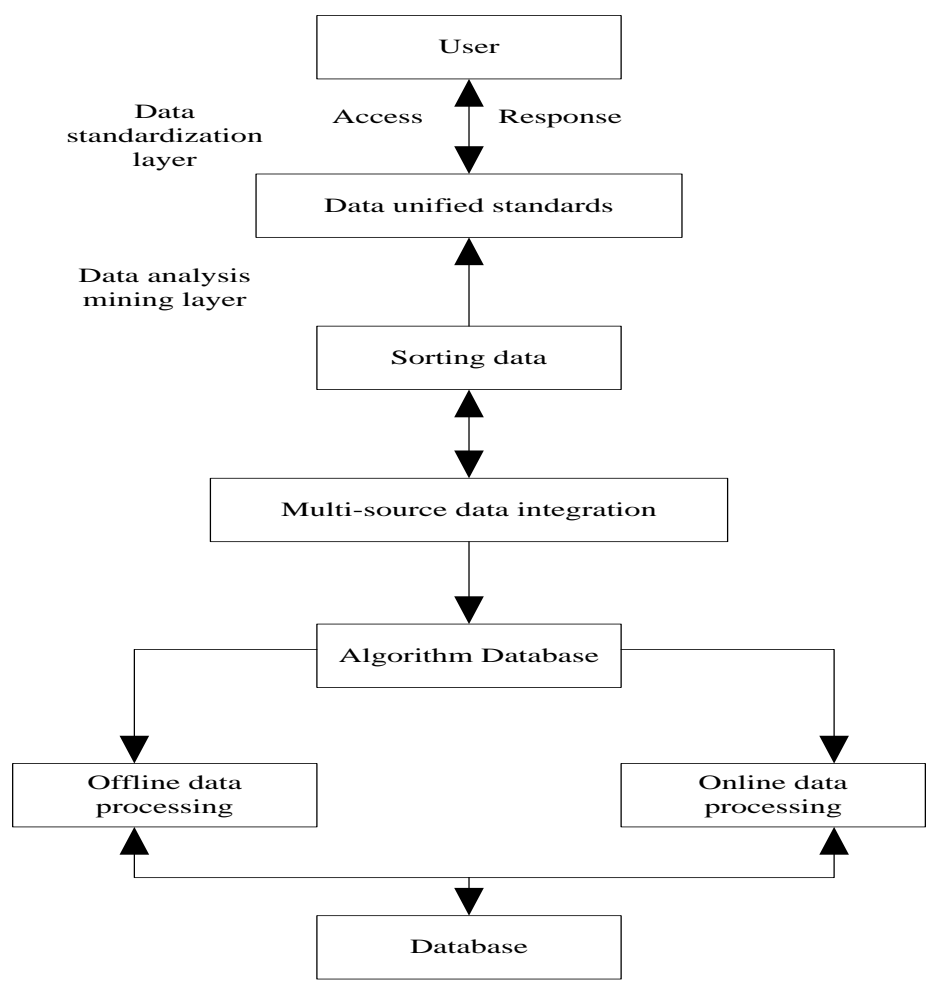

Fig.3. Database Resource Processing Architecture

In the face of rapidly increasing complex data, online education database uses network function virtualization architecture for modern data management, supporting all data types [7], such as files, pictures, videos, blogs, click streams and geospatial data, and the network function virtualization architecture is used to store the data in the data center, keep the data updated in real time, realize data sharing, analysis, discovery, integration and optimization, and improve the data value. Using the advantage of load balance, we can effectively and transparently expand the bandwidth of network devices and servers, increase the throughput of multimedia online education, strengthen the network data processing capacity of the platform, and improve the flexibility and availability of services. In the face of a large number of concurrent access or data flow of users, it can be shared to multiple devices for processing, reducing the waiting response time of teachers and learners; at the same time, parallel processing is done, and the processing results are summarized and returned to the online 
interactive platform, which greatly improves the processing capacity of the platform system. Offline data is the information resource of various databases accessed by users, and it is the user access information and behavior information collected from the server, the client [8], and the proxy server. The big data technology is used to process the data and clear the unnecessary data. The clustering and classification algorithms are used to analyze the pattern of the processed data. The sample data resources are established to prepare for the data flow mining and analysis. Online data is due to the dynamic and large flow characteristics of data flow. When data flow mining is realized, the network function virtualization architecture is used to mine association rules, classification and clustering with less memory and faster processing speed. The structure of user registration module data table and the meaning of fields are shown in Table 4.

Table 4. Structure of User Registration Module Data Sheet and Significance of Fields

\begin{tabular}{ccc}
\hline Serial number & Field Name & Chinese description \\
\hline 1 & ID & Primary key \\
2 & YH & username \\
3 & XM & actual name \\
4 & GK & Do you want to make your name public \\
5 & XB & gender \\
6 & MM & identification number \\
7 & SFZH & E-MAIL \\
8 & EMAIL & date of birth \\
9 & CSRQ & contact number \\
10 & LXDH & contact address \\
11 & LXDD & Postal code \\
12 & YB & Educational level \\
13 & WHCD & Working unit \\
14 & RZDW & Occupation \\
15 & ZW & Health status \\
16 & JKZK & time of participation in work \\
17 & GZSJ & Graduated school \\
18 & BYXX & Majors studied \\
19 & SXZY & Remark \\
20 & BZ & Creation date \\
21 & CJRQ & status \\
22 & ZT &
\end{tabular}


Integration data is to take offline data as the reference of sample database, analyze online data, timely and effective feedback results, and update resource analysis results in time with the passage of time and the change of users' demand for information resources. Through the data mining process [9], we can filter, analyze and integrate data, establish multi resource classification results, make decisions according to different needs of users, and form indexes to provide convenience for users to access and use services. The integration of data is mainly to prepare for the integration of users, analyze the similarity of users' information resources, classify similar users and allocate similar information resources. According to the four dimensions of service requirements of online learners, including basic information, learning style, learning satisfaction and learning perception, the customized service, personalized service and accurate service can be realized, which is convenient for users to extract the resources they need. Finally, according to the different authorization, the resources needed by users are standardized and uploaded to the server.

\subsection{Multimedia Online Education Management System}

By analyzing the attribute data of students in online courses, we can build a reasonable teaching management system, guide teachers to focus on the students who are not active and have poor learning autonomy, so as to help teachers to teach pertinently. Because there are many attributes of students, the eigenvalues also show diversified distribution characteristics, and the artificial sample analysis is time-consuming, laborious and low accuracy, this paper uses the network function virtualization architecture to build the multimedia online teaching management system. The specific method is to use a large number of student data as samples, extract the eigenvalue data and use the eigenvalue data as the learning data of the neural network. After the training of the network function virtualization architecture, it can automatically output the criteria for students' learning attention, so as to help build a reasonable teaching management system [10]. Because the model is a typical multi parameter nonlinear model, the RBF network with good nonlinear approximation performance is selected, that is, radial basis function network. The network has three layers of structure: input layer, hidden layer and output layer. Combined with the analysis of the previous section, the eigenvalues of students' gender, classroom activity, forum activity, homework quality and autonomous learning degree are taken as the input of RBF network, and the attention degree of students is taken as the output of RBF network. The input neuron and output neuron of the established network are 5 and 1 respectively, and the network topology is shown in Figure 4. 


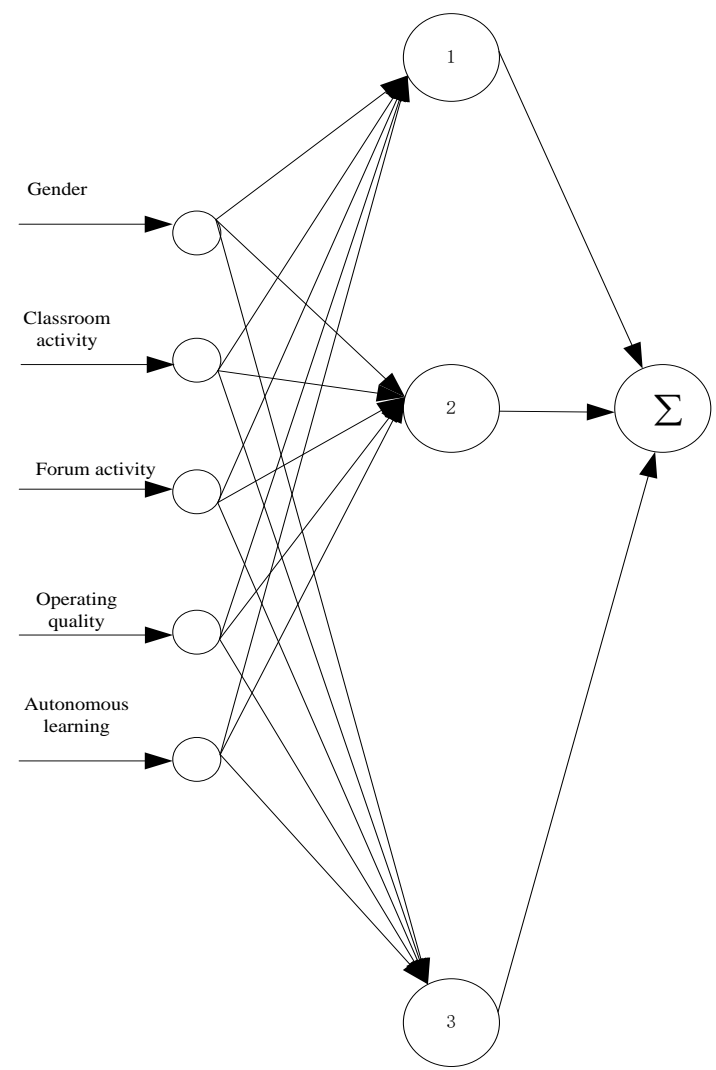

Fig. 4. Multimedia Online Education Management System Network Topology

It can be seen from Figure 4 that the network topology of the multimedia online teaching management system is mainly completed by courseware activities, independent learning, forum activities, etc. on the platform of three-tier architecture, the function module of customer management is realized by using programming technology, function business: online review management module, course dynamic management module, resource download management module, system login interface module Performance management and attendance management module, user password modification interface module, student information management module, teacher information management module, online teaching management module, etc. According to the above definition, the multimedia online education management based on network function virtualization architecture is completed.

\section{Experiment}

In order to verify the effectiveness of a multimedia online education management method based on network function virtualization architecture, and to ensure the rigor of the experiment, compare the traditional method with the management effect of the design method. 


\subsection{Experimental data}

Randomly selected experimental data from a multimedia online education management platform, randomly selected 10 students as the experimental objects, and compared the results of students after two multimedia online education management methods.

In order to record the students'learning situation in real time, the experimental platform is set up, as shown in figure 5 .

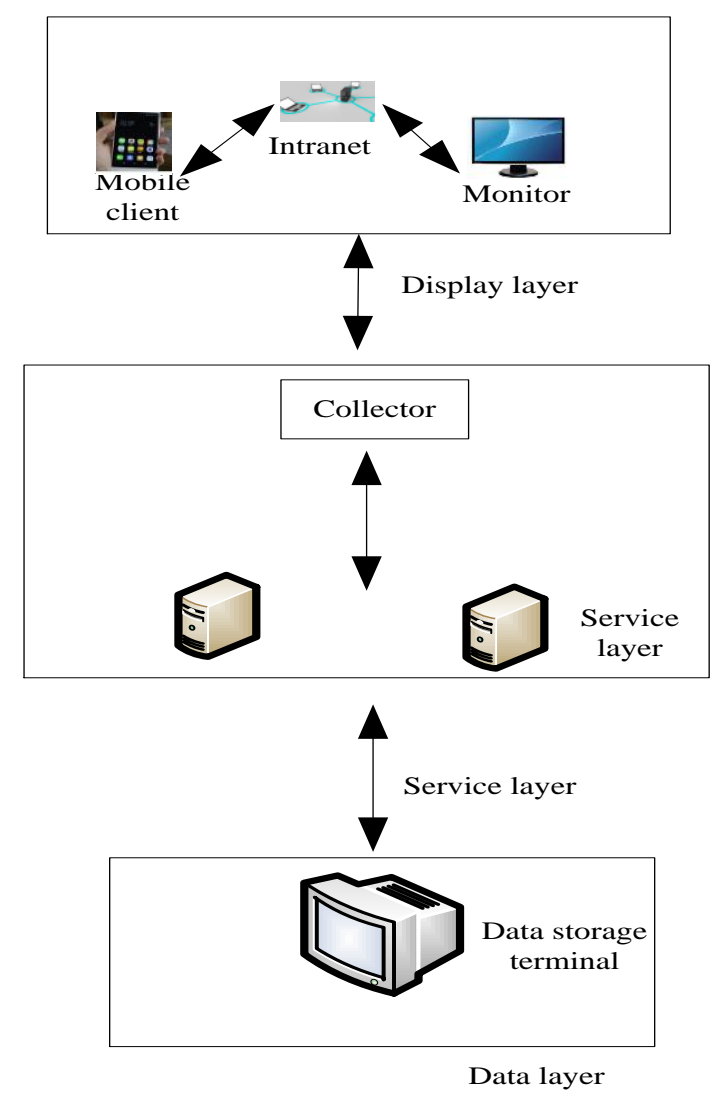

Fig.5. Experimental Platform

In the above experimental platform, the display shows the experimental situation, and the server provides network support for the experiment.

\subsection{Analysis of experimental results}

The traditional multimedia online teaching management method is compared with the multimedia online education management method based on the network function virtualization architecture designed in this paper. The comparison results of the learning achievements after the management are shown in Table 5. 
Table 5. Experimental results

\begin{tabular}{ccc}
\hline $\begin{array}{c}\text { Student } \\
\text { serial } \\
\text { number }\end{array}$ & $\begin{array}{c}\text { Traditional management } \\
\text { methods (learning scores / } \\
\text { points after management) }\end{array}$ & $\begin{array}{c}\text { The design management method (learning score / } \\
\text { point after management) }\end{array}$ \\
\hline 1 & 58 & 90 \\
2 & 45 & 98 \\
3 & 78 & 96 \\
4 & 58 & 95 \\
5 & 63 & 93 \\
6 & 67 & 98 \\
7 & 75 & 97 \\
8 & 77 & 96 \\
9 & 80 & 93 \\
10 & 75 & 94 \\
\hline
\end{tabular}

Analysis of the above results shows that among the 10 students, the learning performance of the multimedia online education management method based on the network function virtualization architecture is significantly higher than that of the traditional management method. In the comparison of the results of the second student, the difference between the two management methods is the biggest, 53 points and 13 points. Therefore, through the above experiments, it can be proved that the multimedia online education management method based on the network function virtualization architecture can improve students' learning performance, meet the needs of multimedia online education management, and provide some help for other multimedia online education management.

\section{Conclusion}

In the current big data environment, the development mode of the education industry has changed dramatically. With online teaching, students can contact experts and scholars around the world, expand the scope of knowledge and expand their thinking. At present, the application of big data in the education industry is not comprehensive, and the attention of teachers and students is far from enough. Online teaching is only limited to homework and preview, which is a waste of educational resources. At the same time, after the traditional management method of multimedia online education, the learning achievement is low. Therefore, a management method of multimedia online education based on the virtual structure of network function is designed. The experiment shows that the students' performance after the design method management is higher than that after the traditional method management. Because the teaching management system can automatically analyze the 
learning state of students according to the data information of students, so that students can be classified according to the degree of attention they should receive. Teachers can carry out targeted teaching according to this classification, so as to improve the teaching effect.

\section{References}

[1] Huang Aihua. Evolution,research and transformation of the teaching mode in higher education [J]. Journal of Higher Education Management, 2017, 11(1):92-96.

[2] Huang Aihua.On the Current Situation and Improvement Strategies of Online Learning for Higher Vocational College Students [J]. Vocational and Technical Education, 2017, 38(14):36-40.

[3] Pan Xiaoyan, Jiang Jiaqiong, Mo Lan,et al. A Comparative Study on the "Quality Matters Rubric Standards" in the U.S. and the Evaluation Index System of Excellent Online Open Course in China [J]. Journal of Educational Science of Hunan Normal University, 2019, 18(3):105-110.

[4] Zheng Li, Wang Jianyu. Analysis of Satisfaction for the Teaching Quality Based on Fuzzy Data Mining [J]. Mathematics in Practice and Theory, 2018, 48(18):106-116.

[5] Chen Jinyin, Wang Ye, Chen Jinye,et al. Design and Research on Intelligent Teaching System Based on Deep Learning [J]. Computer Science, 2019, 46(1):550-554.

[6] Liu Ya, Zeng Xianlu, Hu Xin, et al. Construction and Teaching Practice of the Open Online Courses of Principles of Chemical Engineering [J]. Chinese Journal of Chemical Education, 2018, 39(24):7-14.

[7] Xiao Xiaoying. The Development Trend of Adult Education in the MOOC Era [J]. Adult Education, 2018, 38(1):28-30.

[8] Wang Bimei, Cao Fangfang. On the Construction of Evaluation Index System for Science Teachers' Teaching Competencies Based on Delphi-AHP Approach [J]. Contemporary Education and Culture, 2019, 11(3): 45-53.

[9] Sun Chen, Bi Jun, Zheng Zhilong,et al. MicroNF: a microservice-based hybrid framework for NFV [J]. Journal on Communications, 2019, 40(8): 54-59.

[10] Zhou Zhicheng.The Dilemma in the Reform of Academic Evaluation System in Colleges and Universities and the Deduction of Dynamic Equilibrium Model of Academic Utility [J]. Fudan Education Forum, 2019, 11(3): 38-45. 